Draft Version March 6, 2018

Preprint typeset using LTEX style emulateapj v. 2/16/10

\title{
INFERENCE ON ACCRETION FLOW DYNAMICS USING TCAF SOLUTION FROM THE ANALYSIS OF SPECTRAL EVOLUTION OF H 1743-322 DURING 2010 OUTBURST
}

\author{
Santanu Mondal ${ }^{1}$, Dipak Debnath ${ }^{1}$, Sandip K. Chakrabarti ${ }^{2,1}$ \\ (Dated: Received: 2014 January 16; Accepted: 2014 March 5) \\ Draft version March 6, 2018
}

\begin{abstract}
We study accretion flow dynamics of Galactic transient black hole candidate (BHC) H 1743-322 during its 2010 outburst by analyzing spectral data using Two Component (Keplerian and sub-Keplerian) Advective Flow (TCAF) solution, after its inclusion in XSPEC as a local model. We compare our TCAF solution fitted results with combined disk black body and power-law model fitted results and find a similar smooth variation of thermal (Keplerian or disk black body) and non-thermal (power-law or sub-Keplerian) fluxes/rates in two types of model fits. For a spectral analysis, $2.5-25 \mathrm{keV}$ spectral data from RXTE PCA instrument are used. From the TCAF solution fit, accretion flow parameters, such as Keplerian rate, sub-Keplerian rate, location of centrifugal pressure supported shock and strength of the shock are extracted, thus providing a deeper understanding of accretion process and properties of accretion disks around BHC H 1743-322 during its X-ray outburst. Based on the halo to disk accretion rate ratio (ARR), shock properties, accretion rates and nature of quasi-periodic oscillations (QPOs, if observed) entire outburst is classified into four different spectral states, such as, hard, hard-intermediate, soft-intermediate, and soft. From time variation of intrinsic flow parameters it appears that their evolutions in decline phase do not retrace path of rising phase. Since our current model does not include magnetic fields, spectral turnover at energies beyond 500-600 keV cannot be explained.

Subject headings: stars: individual (H 1743-322); stars: black holes; accretion, accretion discs; shock waves; radiation: dynamics
\end{abstract}

\section{INTRODUCTION}

Galactic transient black hole candidates (BHCs) are very interesting objects to study because these sources generally show evolutions in their temporal and spectral properties during their outburst phases, which are strongly correlated to each other. In last two decades, especially after the launch of Rossi X-ray Timing Explorer (RXTE), our understanding on BHCs has progressed significantly, but not to the extent that we can visualize how the flow configuration and properties are changing within short time scales, say, less than a day. In general, it has been found that during outbursts of a BHC, four basic spectral states, namely, hard, hard-intermediate, softintermediate, and soft are observed (see, Nandi et al., 2012 and references therein). Detailed discussions on the evolution of the temporal and spectral properties of different BHCs during their outbursts were made by several groups (see for e.g., McClintok \& Remillard, 2006; Belloni et al., 2005, Nandi et al., 2012). Different branches of hardness intensity diagram (HID) (Maccarone \& Coppi, 2003; Belloni et al., 2005, etc.) are also found to be related to different spectral states.

It is well established that a standard Keplerian disk (Shakura \& Sunyaev, 1973)cannot explain full X-ray spectrum from black hole candidates and one necessarily requires a second component, namely, the so-called 'Compton' cloud (Sunyaev \& Titarchuk, 1980, 1985), to produce the powerlaw part of the spectrum. There are speculations regarding origin and nature of this Compton cloud which range from a magnetic corona (Galeev, Rosner \& Viana, 1979) to a hot gas corona over the disk (Haardt \& Maraschi, 1993; Zdziarski

\footnotetext{
santanu@csp.res.in; dipak@csp.res.in; chakraba@bose.res.in

${ }^{1}$ Indian Center for Space Physics, 43 Chalantika, Garia St. Rd., Kolkata, 700084, India.

${ }^{2}$ S. N. Bose National Centre for Basic Sciences, Salt Lake, Kolkata, 700098, India.
}

et al., 2003). Observational evidences show that while both the components must be dynamic, one component moves faster (e.g., Smith, et al. 2002; Soria, et al., 2001; Wu, et al. 2002; Cambier \& Smith, 2013) very much like the lowangular momentum, sub-Keplerian transonic flow component as incorporated in Chakrabarti \& Titarchuk (1995, hereafter CT95). In Two Component Advective Flow (TCAF) solution, Chakrabarti and his collaborators, even before the RXTE was launched, envisaged that Compton cloud is actually the inefficiently radiating transonic flow (Chakrabarti, 1990) having very low, or sub-Keplerian angular momentum. Matter in this accretion flow becomes hot close to the black hole where the centrifugal pressure starts dominating and an accretion shock may or may not form depending on whether or not the Rankine-Hugoniot shock conditions are satisfied (CT95; Chakrabarti, 1997, hereafter C97). Recently, Mondal \& Chakrabarti (2013), and Giri \& Chakrabarti (2013) showed that a self-consistent and stable transonic solution exists which supports the solution envisaged by CT95.

The stellar mass BHC $\mathrm{H} 1743-322$ is very intriguing, because in the last decade after its re-discovery in 2003 (Revnivtsev et al. 2003), it showed several X-ray outbursts in regular intervals of $1-2$ years. This source was in a quiescent state for a long time - only a couple of X-ray activities by EXOSAT in 1984 (Reynolds, 1999) and by TTM/COMIS onboard Mir-Kvant in 1996 (Emelyanov et al., 2000) were reported after its first detection in Aug-Sep, 1977, with ArielV All-Sky Monitor (Kaluzienski \& Holt, 1977) and HEAO1 satellite (Doxsey et al., 1997). This Low Mass X-ray Binary $(\mathrm{LMXB})$ system is located at R.A. $=17^{h} 46^{m} 15^{s} .61$ and Dec. $=-32^{\circ} 14^{\prime} 00^{\prime \prime} .6$ (Gursky et al., 1978). Mass of this BHC has not been dynamically confirmed yet, although (Pétri, 2008 ) predicted that its mass is in between $9 M_{\odot}$ to $13 M_{\odot}$ with their high frequency QPO model. Steiner et al. (2012) has confirmed its distance $D=8.5 \pm 0.8 \mathrm{kpc}$, disk inclination an- 
gle $\theta=75^{\circ} \pm 3^{\circ}$, and spin $\left(-0.3<a_{*}<0.7\right.$ with a $90 \%$ confidence).

Recently, in 2010, H 1743-322 was again found to be active in X-rays (Yamaoka et al., 2010) with a similar characteristics of temporal and spectral evolutions as observed in other transient BHCs (Debnath et al., 2008, 2010; Nandi et al., 2012 and references therein). The outburst was observed for a short time period of around two months and RXTE had a full coverage of this source on a daily basis. In Debnath et al. (2013; hereafter Paper-I), a detailed study of the temporal and spectral properties of the source during its two successive outbursts (2010 \& 2011) using RXTE/PCA archival data are presented. In Paper-I, the spectral properties of the source were studied with a combination of conventional thermal (disk black body) and non-thermal (power-law) model components. In order to understand more realistic picture of the accretion flow dynamics, one needs to study spectral properties with a more physical model (such as TCAF), which would enable one to extract actual physical parameters of the accretion flow. In this paper, we study important physical properties, such as time variation of accretion rates of Keplerian and sub-Keplerian components, shock (when present) location and strength of the flow around $\mathrm{H}$ 1743-322 during its 2010 outburst by analyzing spectral properties using TCAF solution. Based on spectral classification method as defined in Debnath, Mondal \& Chakrabarti (2014a), four basic spectral states are also observed during the entire period of this outburst; which also form a hysteresis loop, similar to the HID, where different spectral states belong to different branches of diagram (see. Fig. 4).

This Paper is organized in the following way: in the next Section, we discuss observation and data analysis procedures using HEASARC's HeaSoft software package. In $\S 3$, we present results of spectral analysis using TCAF based model fits file and variation of different flow parameters with observational results. Finally, in $\$ 4$, we present a brief discussion and make our concluding remarks.

\section{OBSERVATION AND DATA ANALYSIS}

We analyze data of 26 observational IDs starting from 2010 August 9 (Modified Julian Day, i.e., MJD = 55417) to 2010 September $30(\mathrm{MJD}=55469)$. We carry out data analysis using FTOOLS software package HeaSoft version HEADAS 6.12 and XSPEC version 12.7. For generation of source and background '.pha' files and spectral fitting using TCAF solution, we use same method as described in Debnath, Mondal \& Chakrabarti (2014a, hereafter DMC14), and Debnath, Chakrabarti \& Mondal (2014b, hereafter DCM14).

The $2.5-25 \mathrm{keV}$ PCA background subtracted spectra are fitted with TCAF based model fits file. To achieve the best fit, a Gaussian line of peak energy around $6.5 \mathrm{keV}$ (iron-line emission) is used. For the entire outburst, we keep hydrogen column density $\left(\mathrm{N}_{H}\right)$ fixed at $1.6 \times 10^{22}$ atoms $\mathrm{cm}^{-2}$ for absorption model wabs and assume a $1.0 \%$ systematic error (PaperI). After achieving best fit based on reduced chi-square value $\left(\chi_{\text {red }}^{2} \sim 1\right)$, 'err' command is used to find $90 \%$ confidence error values for the model fitted parameters. We have not included HEXTE data since the rocking mechanism stopped in 2010 and thus it is difficult to subtract the background.

For a spectral fit using the TCAF based model, one needs to supply a total of six input parameters: $i)$ Keplerian rate $\left(\dot{m}_{d}\right.$ in Eddington rate), $i i)$ sub-Keplerian rate ( $\dot{m}_{h}$ in Eddington rate), iii) black hole mass $\left(M_{B H}\right)$ in solar mass $\left(M_{\odot}\right)$ unit, $\left.i v\right)$ location of the shock $\left(X_{S}\right.$ in Schwarzschild radius $\left.r_{g}=2 G M / c^{2}\right)$, $v$ ) compression ratio $(R)$ of the shock, and $v i)$ the model normalization value (norm) of $\frac{R_{z}}{4 \pi D^{2}} \sin (i)$, where ' $R_{z}$ ' is the effective height of the Keplerian component in $\mathrm{Km}$ at the preshock region, ' $D$ ' is the source distance in $10 \mathrm{kpc}$ unit and ' $i$ ' is the disk inclination angle. In order to fit a black hole spectrum with TCAF model in XSPEC, we generate model fits file (TCAF.fits) using theoretical spectra generating software by varying five input parameters in CT95 code and then include it in XSPEC as a local additive model. Detail description of the range of input parameters and generation procedure is mentioned in DCM14 and DMC14. In this Paper, for the spectra of entire outburst data, we consider mass of the black hole as $11.4 \pm 1.9 M_{\odot}$ (Debnath et al., 2014c).

\section{RESULTS}

Accretion flow dynamics during an outburst phase of transient BHCs can be well understood by model analysis of spectral and temporal behavior of the source. These behaviors have already been discussed in Paper-I though the analysis was made using a combination of disk black body (DBB) and power-law (PL) model components which give only the gross properties of the disk. TCAF based model goes one step further in extracting the detailed flow parameters, such as two disk rates and shock properties. Furthermore, this makes the boundary of the states more well-defined. Thus, for accretion dynamics, we need to use the TCAF based model fits file.

\subsection{Results of Spectral Data Fitted by TCAF Solution}

Figure 1 shows variation of X-ray intensities, QPO frequencies along with model fitted parameters. In Fig. 1a, variation of background subtracted RXTE PCA count rate in $2-25 \mathrm{keV}$ (0 - 58 channels) energy band with day (MJD) is shown. Figs. $1 \mathrm{~b} \& 1 \mathrm{c}$ show variations of combined DBB and PL model fitted total spectral flux (flux contributions for DBB and PL model components are calculated by using convolution model 'cflux' technique after fitting spectra with combined model components) in $2.5-25 \mathrm{keV}$ energy band and TCAF model fitted total accretion rates (combined Keplerian disk and sub-Keplerian halo rates) in the same energy band. In Fig. 1d, variation of Accretion Rate Ratio (ARR, defined to be ratio of sub-Keplerian halo rate $\dot{m}_{h}$ and Keplerian disk rate $\left.\dot{m}_{d}\right)$. Variations of TCAF model shock locations $\left(X_{s}\right)$ and compression ratios are shown in Fig. 1e \& 1f respectively. Observed QPO frequencies are shown in Fig. 1g. Depending on variation of $\mathrm{ARR}$ and nature (shape, frequency, $Q$ value, $\mathrm{rms} \%$ etc) of QPOs (when observed), four different spectral states such as hard (HS), hard-intermediate (HIMS), softintermediate (SIMS), soft (SS) could be identified during the entire outburst of H 1743-322 (for details see, DMC14). We observe that spectral transitions between these states occur approximately at the same day as reported in Paper-I (marked with vertical dashed lines in Figs. 1-2), where spectral classifications were done on the basis of degree of importance of DBB and PL model components (fluxes) and properties of QPOs. We see that the TCAF model fits well for all four spectral states (model fitted $\chi_{\text {red }}^{2}$ varies $\sim 0.9-2$ ) obeying spectral results of combined DBB \& PL. As of DMC14 for 2010-11 GX 339-4 outburst, here we also find two surges in ARR at the boundaries of hard-intermediate states, i.e., from hard to hard-intermediate state during rising phase or from hard-intermediate to hard state during declining phase of the outburst) transition days. Note that from the TCAF fit, sum of the accretion rates appears to be almost constant in the hard 
states, both before and after the outburst. At the onset of the soft-intermediate state, the total rate rises very sharply. This continues till soft-intermediate state ends. The rate decays monotonically till the end of the hard intermediate state. This will be clear from Fig. 2, where we show comparative variations of the combined DBB and PL model fitted DBB and PL fluxes along with TCAF model fitted Keplerian disk $\left(\dot{m}_{d}\right)$ and sub-Keplerian halo $\left(\dot{m}_{h}\right)$ rates with day (MJD). It is important to note that these two variations are going hand in hand. We shall return back to discussions on Figs. 1(e-g) later.

As a further consistency check of TCAF, we may ask the following: since the power-law component is due to scattering of intercepted black body photons by Compton cloud, which, according to TCAF, is produced by radiatively inefficient, low angular momentum transonic halo component, could variations of PL flux and $\dot{m}_{h}$ be similar? From the Figure 2(c-d) the similarity is obvious. Indeed the behavior shown in Fig. 2 justifies us using the more physical model, i.e., TCAF which directly gives the intrinsic flow parameters. Furthermore, this also shows that the halo rate plays a major role in deciding the spectral properties of the flow. However, at times, the two rates vary independently as is obvious from Fig 1d. This will be discussed later.

In Table 1, TCAF model fitted parameters along with frequency of primary observed QPOs (if present) are mentioned. In Fig. 3, unabsorbed theoretical model spectra for seven states in $0.005-1000 \mathrm{keV}$ energy range, selected from different spectral states of the outburst as marked in the Table, are shown. Note that relatively harder states clearly show spectral turnover above $\sim 300 \mathrm{keV}$. It is also to be noted that since present model of TCAF does not include magnetic fields explicitly, inverse Comptonization of non-thermal photons produced in the post-shock region could not be included and thus turnovers which may occur at much higher energies in sources such as Cyg X-1 (Zdziarski 2000; Zdziarski et al. 2001; Chakrabarti \& Mondal, 2006) cannot be fitted with the fits file generated using the current TCAF model.

\subsection{Evolution of Spectral and Temporal Properties during the Outburst}

Detailed temporal and spectral properties of this candidate are discussed by several authors on the basis of whether QPO observation was made or not (Belloni et al. 2005; McClintock et al. 2009; Paper-I). However, since we have physical parameters on each day, it may be instructive to check if there is any physical way to differentiate one spectral state from another. In Figs. $1 \mathrm{~d}$ and $1 \mathrm{~g}$, we see variations of ARR and QPO frequencies with time in MJD. It seems that ARR, total flow/accretion rate $\left(\dot{m}_{d}+\dot{m}_{h}\right)$, shock locations, compression ratios, etc. in conjunction with QPOs provide a better understanding on the classification of spectral states.

(i) Hard State in the Rising phase: For the first 3 days of RXTE observation (from MJD = 55417.29 to 55419.11), source was in a hard state with increasing total flux and nonthermal PL flux (or, equivalently, sub-Keplerian halo rate; see, Figs. 1-2). During this phase, halo rate is increasing faster than disk rate as the infall time of the halo is shorter than that of the disk. ARR is monotonically increasing and reached a value of 3.33, maximum for the entire outburst. QPO frequencies are observed to be increasing monotonically from $0.919 \mathrm{~Hz}$ to $1.045 \mathrm{~Hz}$ (see, Paper-I). We define the day of maximum ARR as the transition day from the hard to hardintermediate spectral state.

(ii) Hard-Intermediate State in the Rising phase: The source was in this state for the next $\sim 5$ days after the transition day $(\mathrm{MJD}=55419.11)$. During this phase, QPO frequency continues to increase monotonically till it reached from $1.045 \mathrm{~Hz}$ on MJD $=55419.11$ to $4.796 \mathrm{~Hz}$ on MJD $=55424.06$. Supply of sub-Keplerian matter is continued at the same rate and a part of it is converted to Keplerian matter due to viscous effects, thereby increasing its rate. Consequently, ARR is decreased. We define the end of hard-intermediate state up to time when the episode of constant ARR begins.

(iii) Soft-Intermediate State in the Rising phase : The constancy of ARR lasted till total rate suddenly jumps. Both disk and halo rates along with PCA count rapidly increased till QPOs last. On this day (MJD = 55425.16), the observed QPO frequency $(3.558 \mathrm{~Hz})$ did not increase as before. This date of the highest total rate and total PCA counts ends the soft-intermediate state and usher the object to the next, i.e., soft state.

(iv) Soft State: The source is observed at this state for the next $\sim 26$ days (up to MJD $=55450.34$ ), where the spectra were mostly dominated by thermal photons. Shock location is very close to the black hole, but is not oscillating as the resonance oscillation condition is not fulfilled in presence of rapid cooling. As a result, the QPO is absent. The continuous drainage of Keplerian and sub-Keplerian matter reduced the total rate monotonically, while keeping ARR roughly constant. This state continues till the drainage is just enough to bring back resonance condition for the oscillation of the CENtrifugal pressure supported Boundary Layer (CENBOL) whose outer boundary is the shock (see, Molteni, Sponholz and Chakrabarti, 1996; hereafter MSC96) and QPOs reappear. ARR became almost same as that on the date QPO was last observed in the Soft intermediate state. On MJD = 55451.17, a state transition is observed.

(v) Soft-Intermediate State in the Declining phase : For the following $\sim 5$ days, the source was observed in this state. During this period, the total flux (DBB+PL, PCA count rate and $\dot{m}_{d}+\dot{m}_{h}$ ) is almost constant, though individually the rates fluctuate. Sporadic QPOs of $\sim 2 \mathrm{~Hz}$ are observed during this spectral state. Sporadic phase of QPO is over on MJD $=55455.44$, which signifies the end of this state.

(vi) Hard-Intermediate State in the Declining phase : On MJD $=55455.44$, hard-intermediate state starts. After that, continuous QPOs are observed. ARR on this day is roughly the same it was in the last day of hard-intermediate state in the rising phase. Initially, the Keplerian disk is drained more rapidly and thus ARR increases rapidly. Total accretion rate, net PCA count etc. continued to be drained and centrifugal pressure supported shock location started receding (Fig. 1e) as the incoming flow pressure drops. As a result, QPO frequency drops steadily (see, Paper-I). On MJD $=55462.56$, maximum ARR (=2.62) of declining phase is observed, which defines the transition day from hard-intermediate state to hard state.

(vii) Hard State in the Declining phase: Source is observed in this spectral state till the end of the observation of 2010 outburst. In this state ARR value decrease monotonically from 2.62 to 1.50 and observed QPOs also decrease monotonically from $0.74 \mathrm{~Hz}$ to $79 \mathrm{mHz}$ (see, Paper-I). However, the total rate becomes roughly constant as in the rising phase. The rising phase was observed several days after it began. In the declining phase observation continued till PCA count was much lower than that on the first day of the rising phase. The end of hard state concludes the outburst. 


\section{DISCUSSIONS AND CONCLUDING REMARKS}

We analyzed spectral properties of Galactic transient black hole candidate $\mathrm{H}$ 1743-322 during its 2010 outburst using two component advective flow (TCAF) solution based model after its inclusion as a local additive table odel in HEASARC's spectral analysis software package XSPEC. We generate $4 \times 10^{5}$ model spectra using CT95 code to fit with the observational data. Our fits file generation procedure is the same as that presented in DMC14. Flow parameters (Keplerian and sub-Keplerian accretion rates) extracted from TCAF model spectral fit generally match with DBB and PL fluxes (see, Figs. 1-2). Total rate, shock location, QPOs in different spectral states have been observed to behave very reasonably. From these quantities, we have a complete physical picture of when exactly spectral state transitions occur.

In Fig. 4(a-b) we give two Figures where we show variation of derived physical quantities of the flow from our fits. In (a), we plot variation of PCA count rate as a function of ratio $1 / A R R$ and in (b), disk rate as a function of the halo rate for the whole outburst. B, C, D, E, F and G are points where state transitions take place. In (a), segments A-B (black online) and G-H (orange online) show highest ARR locations though PCA counts are different, the rising phase A-B (when first caught) being considerably brighter. Segments B-C (red online) and G-F (cyan online) have roughly similar range of ARR. The pattern of first horizontal and then vertical variation of PCA count in the rising phase is reversed in the decline phase. Segments C-D (green online) and E-F (magenta online) are soft-intermediate states occurring roughly at the same ARR value in the rising phase (sharp rise in PCA count) but rapid oscillation in ARR, though ARR at the edges remain roughly the same. Segment D-E (blue online) is fluctuating, drainage of disk and halo being alternately high. Fig. $4 \mathrm{~b}$ clearly shows that the two rates are independent and they do not always increase or decrease together, except only in soft state. Indeed, sometimes they are horizontal and sometimes vertical. In these cases, only one component changes keeping the other component as constant.

As far as the low frequency QPO behavior is concerned, it can occur only when the cooling time scale roughly agrees with the infall time scale (MSC96). In hard and hardintermediate states this is easily achievable as shocks are formed and Compton cooling time scale is similar to infall time scale from the shock to the inner edge of the disk. In soft and soft-intermediate states (when $\dot{m}_{d}>1$, see Fig. 4b),
CENBOL is cooled down very rapidly and shock may or may not be seen. This is true in both rising and decline phases. In soft-intermediate states QPOs are therefore seen sporadically. When the total rate is maximum, soft intermediate state transits to soft state and QPO is not seen any more. In hard intermediate states, $\dot{m}_{d}$ decreases keeping while $\dot{m}_{h}$ remains roughly a similar number. Thus ARR increases while going from hard-intermediate states to hard states. ARR develops a sharp maximum on the day of transition from hard to hardintermediate state in the rising phase, and the reverse in the decline phase. This does show that QPOs are strongly coupled to cooling properties of the disk.

In the literature, it is in vogue to study the outburst properties using a so-called 'q'-diagram where hardness is plotted with intensity throughout the outburst (Paper-I and references therein). One of the disadvantages of this is that there is no unique definition of hardness and the energy ranges used in the numerator and denominator are rather arbitrary and may be mixtures of soft and Comptonized photons depending on the mass of the black holes. In a TCAF model, we directly extract the accretion rate components and visualize what happens to the object during the outburst (see also, Mandal \& Chakrabarti, 2010). Our direct approach gives more physical insight. Particularly interesting is that the accretion rate ratio plays a very vital role in defining the spectral state transitions.

Earlier we showed (DMC14) how spectral state transitions occur due to variation of Keplerian and sub-Keplerian rates during 2010-11 outburst of Galactic BHC GX 339-4. We showed variation of physical parameters, such as accretion rates, ARR, and shock location and certain behaviors of these parameters at the time of state transitions. From the variation of Keplerian rate in both these cases, we are now convinced that an outburst is triggered due to a sudden rise in viscosity. Viscous time scale brought much of Keplerian disk matter in a time scale of about eight days (MJD 55417 to 55425) in $\mathrm{H}$ 1743-322. Drainage took longer time as this matter is entrained with sub-Keplerian halo. It would be interesting to check if flow dynamics of other outbursting sources also follow a similar trend. Prediction of QPO frequency from TCAF solution fitted shock parameters $\left(X_{S} \& R\right)$, and comparative study with POS model solution (Paper-I) will be published elsewhere.

\section{ACKNOWLEDGMENTS}

S. Mondal acknowledges the support of CSIR-NET scholarship.

\section{REFERENCES}

Belloni, T., Homan, J., Casella, P., et al., 2005, A\&A, 440, 207

Cambier, H.J., \& Smith, D.M., 2013, ApJ, 767, 46

Chakrabarti, S.K., 1990, "Theory of Transonic Astrophysical Flows", World Scientific (Singapore)

Chakrabarti, S.K., 1997, ApJ, 484, 313

Chakrabarti, S. K. \& Mandal, S. 2006, ApJ, 642, L49

Chakrabarti, S.K. \& Titarchuk, L.G., 1995, ApJ, 455, 623

Debnath, D., Chakrabarti, S. K., \& Nandi, A., et al., 2008, BASI, 36, 151

Debnath, D., Chakrabarti, S. K., \& Nandi, A., 2010, A\&A, 520, 98

Debnath, D., Chakrabarti, S. K., \& Nandi, A., 2013, AdSpR, 52, 2143 (Paper-I)

Debnath, D., Mondal, S., \& Chakrabarti, S. K., 2014a, ApJ (submitted) (arXiv:1306.3745) (DMC14)

Debnath, D., Chakrabarti, S. K., \& Mondal, S., 2014b, MNRAS Letters (in press) (DCM14) (arXiv:astro-ph/1402.0989)

Debnath, D., Chatterjee, A., Chakrabarti, S. K., \& Mondal, S., 2014c (in preparation)

Doxsey, R., Bradt, H., \& Fabbiano, G., et al., 1997, IAU Circ., 3113, 1
Emelyanov, A. N., Aleksandrovich, N. L., \& Sunyaev, R. A., 2000, AstL, 26, 297

Galeev, A. A., Rosner, R., \& Vaiana, G. S., 1979, ApJ, 229, 318

Giri, K., \& Chakrabarti, S. K., 2013, MNRAS, 430, $2836 \mathrm{G}$

Gursky, H., Bradt, H., \& Doxsey, R. et al., 1978, ApJ, 223, 973

Haardt, F., \& Maraschi, L., 1993, ApJ, 413, 507

Kaluzienski, L. J., \& Holt, S. S., 1997, IAU Circ., 3099, 3

Maccarone, T. J., \& Coppi, P. S., 2003, MNRAS, 338, 189

Mandal, S. \& Chakrabarti, S. K., 2010, ApJ, 710, L147

McClintok, J. E., \& Remillard, R. A., 2006 (arXiv:astro-ph/0306213)

McClintock, J. E., Remillard, R. A., \& Rupen, M. P., et al., 2009. ApJ, 698, 1398

Mondal, S., \& Chakrabarti, S. K., 2013, MNRAS, 431, 2716

Molteni, D., Sponholz, H. \& Chakrabarti, S.K., 1996, ApJ, 457, 805

Pétri, J., 2008, Ap\&SS, 318, 181

Nandi, A., Debnath, D., \& Mandal, S., et. al., 2012, A\&A, 542, 56

Reynolds, P. S., 1999, ApL\&C, 38, 425

Revnivtsev, M., Chernyakova, M., \& Capitanio, F., et al., 2003, ATel, 132, 1 
Shakura, N. I. \& Sunyaev, R. A., 1973, A\&A, 24, 337

Smith, D. M., Heindl, W. A., \& Swank, J. H., 2002, ApJ, 569, 362

Soria, R., Wu, K., \& Hannikainen, D., et al., 2001, Proc. of workshop on

"X-Ray emission from Accretion onto Black Holes", 65

Steiner, J. F., McClintock, J. E., \& Reid, M. J., 2012, ApJ, 745, L7

Sunyaev, R.A., \& Titarchuk, L. G., 1980, ApJ, 86, 121

Sunyaev, R.A., \& Titarchuk, L. G., 1985, A\&A, 143, 374

Wu, K. et al., 2002, ApJ, 565, 116
Yamaoka, K., Negoro, H., \& Sugizaki, M., et al., 2010, ATel, 2378, 1

Zdziarski, A. A., 2000, in IAU Symp. 195, High Energetic Physical

Processes and Mechanisms for Emission from Astrophysical Plasma, ed.

P. C. H. Martens, S. Tsuruta, \& M. A. Weber (San Francisco: ASP), 153

Zdziarski, A. A., Grove, J. E., Poutanen, J., Rao, A. R., \& Vadawale, S. V., 2001, ApJ, 554, L45

Zdziarski, A. A., Lubinski, P., \& Gilfanov, M., et al., 2003, MNRAS, 342, 355

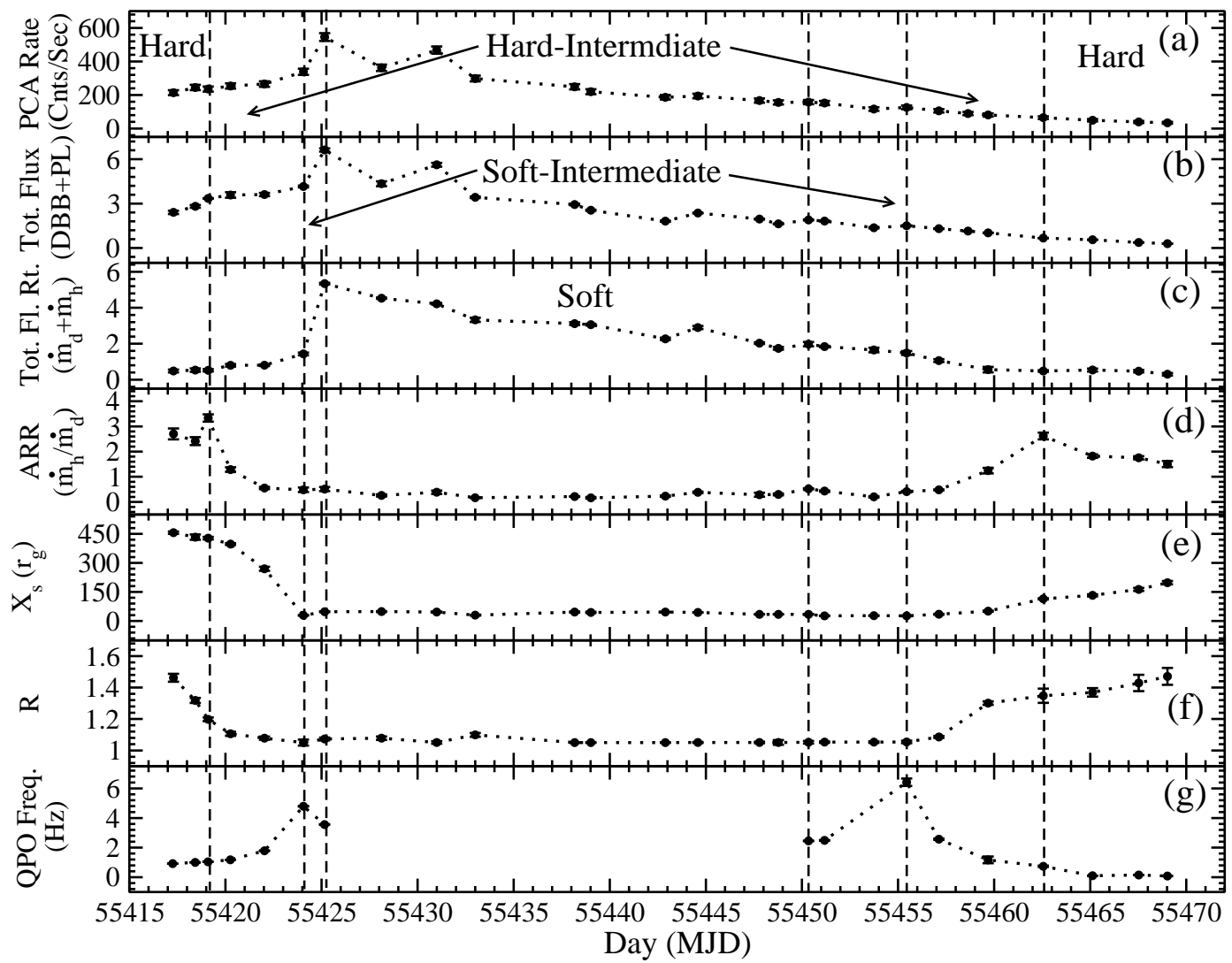

FIG. 1.- Variation of (a) $2-25 \mathrm{keV}$ PCA count rates (cnts/sec), (b) combined disk black body (DBB) and power-law (PL) model fitted total spectral flux in $2.5-25 \mathrm{keV}$ range, (c) TCAF model fitted total accretion rate (sum of Keplerian disk rate $\dot{m}_{d}$ and sub-Keplerian halo rate $\dot{m}_{h}$ ) in the $2.5-25 \mathrm{keV}$ energy band, (d) ARR (i.e., ratio between halo and disk rates) with day (MJD) for the 2010 outburst of H 1743-322 are shown. In bottom three panels, variations of observed QPO frequency in $\mathrm{Hz}(\mathrm{g})$ and shock location (e), compression ratio (f) are shown. Vertical dashed lines are drawn where we believe transitions of different spectral states have actually taken place. 


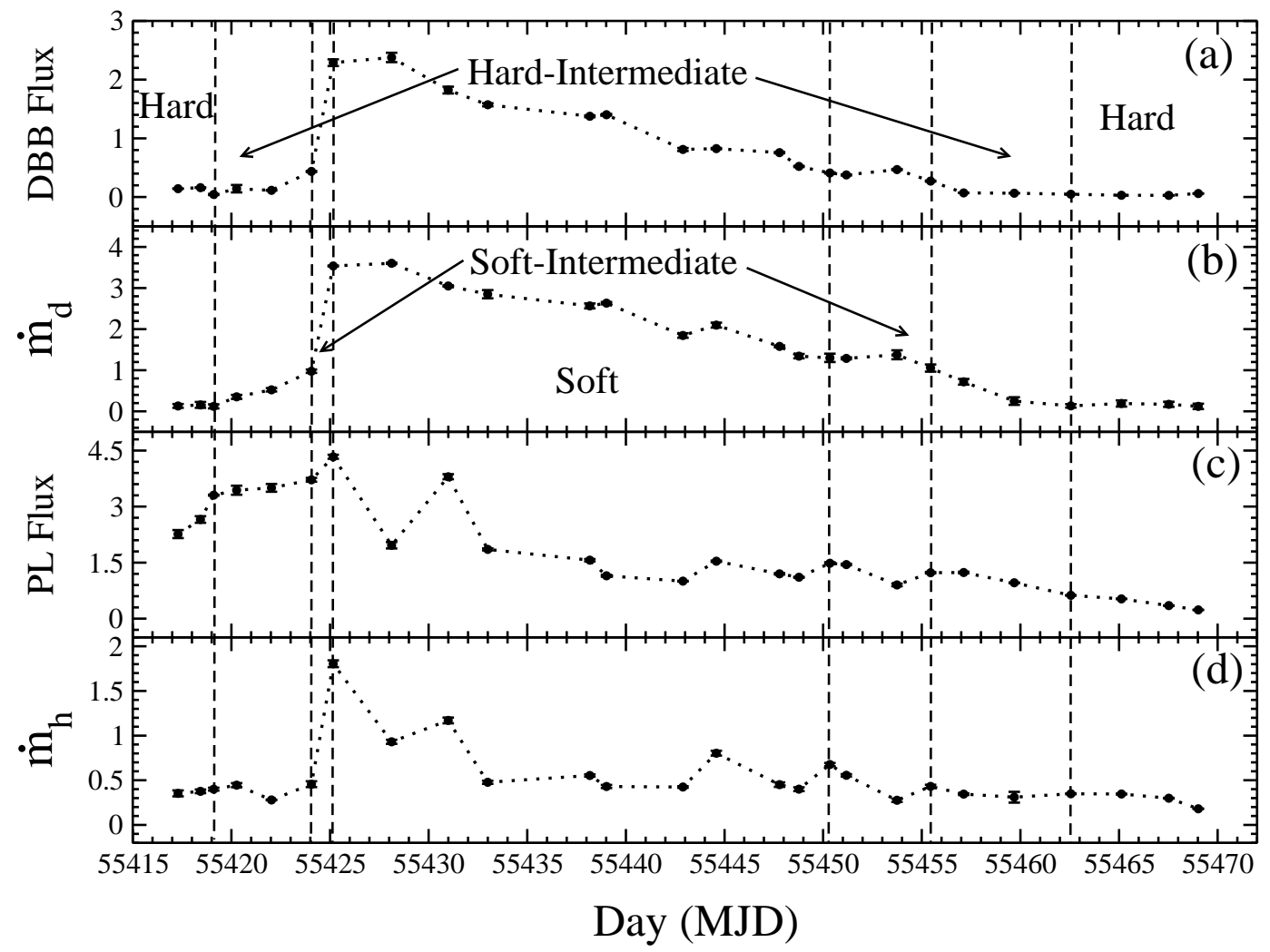

FIG. 2.- Variation of combined disk black body (DBB) and power-law (PL) model fitted DBB spectral flux in $2.5-25$ keV energy range (a) and variation of TCAF model fitted Keplerian disk rate $\left(m_{d}\right)$. Though models are different, these variations appear to be very similar. This is a consistency check for the TCAF solution. In (c), PL spectral flux in same energy range and in bottom panel (d), variation of TCAF model fitted sub-Keplerian halo rate ( $\left.\dot{m}_{h}\right)$ in the same energy band are shown. Though models are different, variations of DBB flux and $\dot{m}_{d}$ or PL flux and $\dot{m}_{h}$ appear to be very similar.

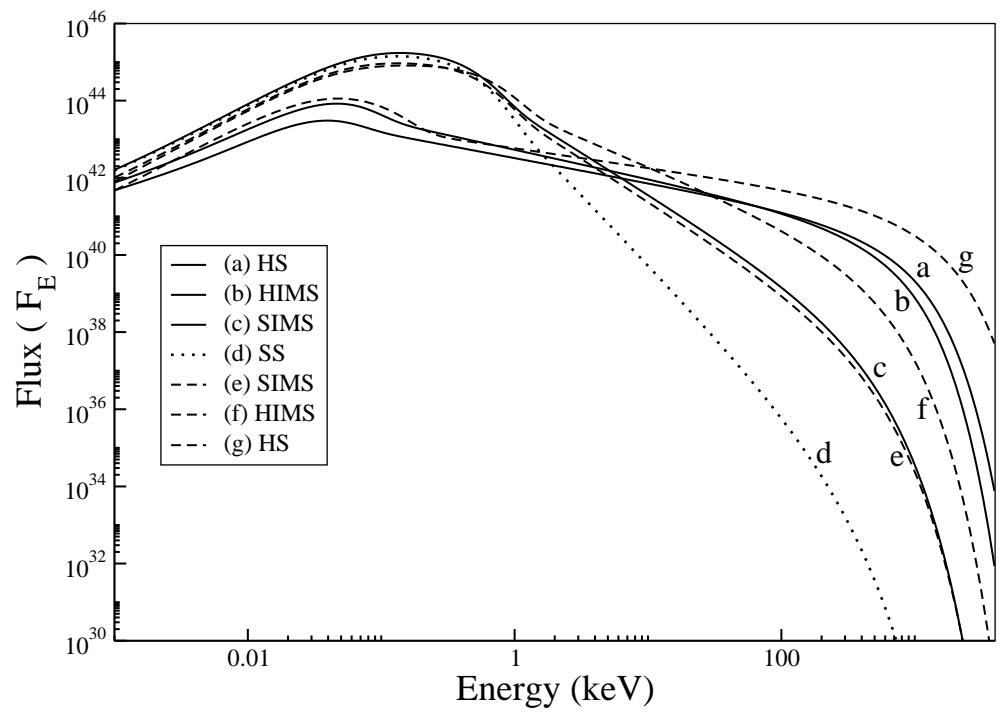

FIG. 3.- Unabsorbed TCAF model generated spectra for four different spectral states, which were used to fit the spectra as marked in Table 1. Solid-line plots $(\mathrm{a}-\mathrm{c})$ are from the rising phase and dashed-line plots (e-g) are from the declining phase of the outburst. Dotted plot (d) shows the spectrum of the soft state. Flux $\left(F_{E}\right)$ is in units of photons $\mathrm{cm}^{-2} \mathrm{sec}^{-1} \mathrm{keV}^{-1}$. 

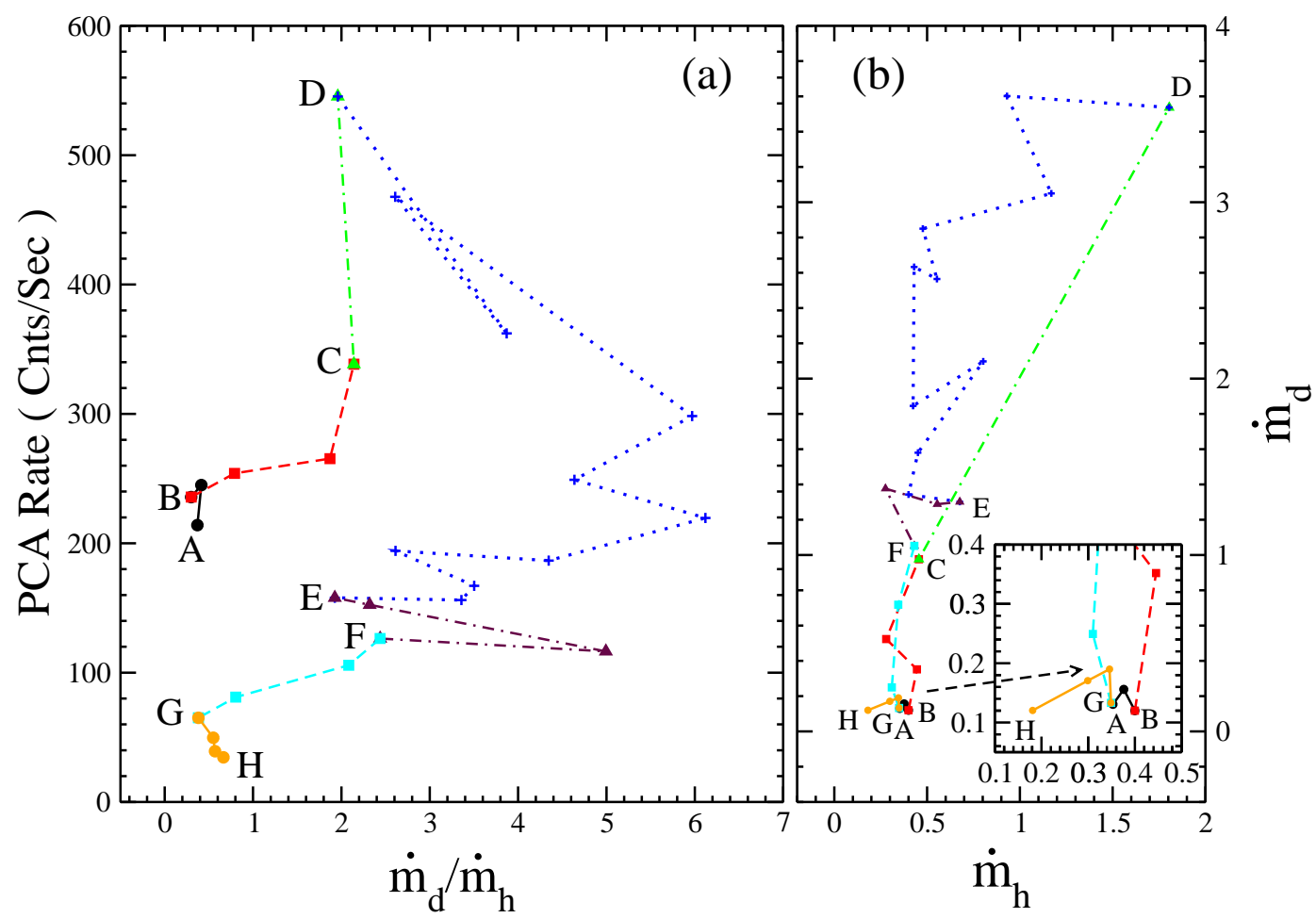

FIG. 4.- (a) Variation of PCA count rate as a function of 1/ARR and (b) disk rate as a function of halo rate (b) for the whole outburst. Transitions from hard to hard intermediate (rising) and vice versa (declining) takes place when ARR is separetely maximum. Soft state begins with the highest value of individual rates. Duration of hard intermediate states in both phases occur at a similar value of ARR. For ARR $\leq 0.5$, QPOs are sporadic or absent.

Table 1

2.5-25 keV TCAF Model Fitted Parameters with QPOs

\begin{tabular}{|c|c|c|c|c|c|c|c|c|c|c|}
\hline Obs. & (2) & (3) & MJD & $\begin{array}{c}\dot{m}_{d} \\
\left(\dot{M}_{E d d}\right) \\
(5)\end{array}$ & $\begin{array}{c}\dot{m}_{h} \\
\left(\dot{M}_{E d d}\right) \\
(6)\end{array}$ & ARR & $\begin{array}{c}X_{s} \\
\left(r_{g}\right) \\
(8)\end{array}$ & (9) & $\begin{array}{c}\text { QPO } \\
(\mathrm{Hz}) \\
(10)\end{array}$ & $\chi^{2} / D O F$ \\
\hline 1 & X-01-00 & 09/08/10 & 5417.29 & $0.131 \pm 0.036$ & $353 \pm 0.023$ & $2.702 \pm 0.218$ & $456.0 \pm 6.15$ & $1.462 \pm 0.025$ & $0.919 \pm 0.004$ & $59.28 / 42$ \\
\hline $2^{a}$ & $01-00$ & $/ 08 / 10$ & 418.43 & $156 \pm 0.052$ & $376 \pm 0.021$ & $2.415 \pm 0.157$ & $33.1 \pm 13.7$ & $1.318 \pm 0.016$ & $1.002 \pm 0.003$ & $3.66 / 42$ \\
\hline 3 & & & & & & 46 & 56 & & & $2.72 / 42$ \\
\hline 4 & & & & & & & & & & $743 / 42$ \\
\hline $5^{b}$ & & & & & & & & & & \\
\hline 6 & & & & & & & & & & \\
\hline 7 & & & & & & & & & & \\
\hline 8 & & & & & & & & & ----- & \\
\hline 9 & & & & & & & & & ----- & \\
\hline 10 & & & & & & & & & ----- & \\
\hline 11 & & & & & & & & & ----- & $/ 45$ \\
\hline $12^{d}$ & & & & & & 30 & & & ----- & $9.60 / 45$ \\
\hline 13 & & & & & & & & & ----- & \\
\hline 14 & & & & & & & & & ----- & $59.33 / 45$ \\
\hline 15 & & & & & & & & & ----- & $34.08 / 44$ \\
\hline 16 & & $9 / 09 / 10$ & & & 1 & $0.298 \pm$ & 65 & & ----- & $1.43 / 45$ \\
\hline 17 & & & & & & & & & $2.454 \pm 0.022$ & $33.70 / 44$ \\
\hline $18^{e}$ & $20-01$ & & & & & & & & $2.489 \pm 0.018$ & $3.51 / 45$ \\
\hline 19 & & & & & & & & & ----- & $9.90 / 45$ \\
\hline 20 & & & & & 8 & & & & $6.417 \pm 0.252$ & $1.57 / 42$ \\
\hline $21^{f}$ & & & & & & & & & & \\
\hline 22 & & & & & & & & & & \\
\hline $23^{g}$ & & & & & & & & & & \\
\hline 24 & & & & & & & & & & \\
\hline 25 & & & & & & & & & 005 & $36.72 / 42$ \\
\hline 26 & Y-28-01 & $30 / 09 / 10$ & 5469.01 & $0.120 \pm 0.065$ & $.181 \pm 0.013$ & $1.504 \pm 0.122$ & $197.9 \pm 8.46$ & $1.471 \pm 0.054$ & $0.079 \pm 0.002$ & $38.72 / 42$ \\
\hline
\end{tabular}

Here $\mathrm{X}=95368-01, \mathrm{Y}=95360-14$ indicate the initial part of the observation Ids, and UT date in $\mathrm{dd} / \mathrm{mm} / \mathrm{yy}$ format.

$\dot{m}_{h}$, and $m_{d}$ represent TCAF model fitted sub-Keplerian (halo) and Keplerian (disk) rates in Eddington rate respectively.

$X_{s}$ (in Schwarzchild radius $r_{g}$ ), and $R$ are the TCAF model fitted shock location and compression ratio values respectively.

Here, frequency of the pricipal QPO in Hz are only presented. DOF means degrees of freedom of the model fit. 